\title{
MicroRNA-302a suppresses cell proliferation, migration and invasion in osteosarcoma by targeting ADAM9
}

\author{
XIAOMING YANG, YAN CUI, FUQIANG YANG, CHAO SUN and XUEJIAN GAO \\ Department of Orthopedics, 89th Hospital of PLA, Weifang, Shandong 261021, P.R. China
}

Received June 23, 2016; Accepted April 24, 2017

DOI: $10.3892 / \mathrm{mmr} .2017 .6975$

\begin{abstract}
Osteosarcoma (OS) is the most frequent malignant primary bone tumor arising from primitive bone-forming mesenchymal cells in children and adolescents. The dysregulation of microRNAs (miRNAs) has been reported in OS, and these aberrantly expressed miRNAs are involved in the initiation and progression of OS. The aim of the present study was to investigate the expression and functions of miRNA-302a (miR-302a) in OS and its underlying mechanism. It was found that the expression of miR-302a was reduced in OS tissues and cell lines. The low expression of miR-302a was significantly correlated with tumor-node-metastasis stage and metastasis. The ectopic overexpression of miR-302a inhibited the proliferation, migration and invasion of OS cells. Bioinformatics analysis showed that a disintegrin and metalloproteinase 9 (ADAM9) was a potential target gene of miR-302a. Subsequently, reverse transcription-quantitative polymerase chain reaction and western blot analyses revealed that miR-302a regulated the expression of ADAM9 at the post-transcriptional level in OS cells. In addition, a luciferase reporter assay demonstrated that miR-302a directly targeted the 3'untranslated region of ADAM9. In clinical OS tissues, the mRNA expression of ADAM9 was upregulated and inversely correlated with the expression of miR-302a. In addition, the effects of ADAM9 knockdown on cell proliferation, migration and invasion were similar to those induced by the overexpression of miR-302a in OS cells. These findings suggested that miR-302a inhibited OS cell growth and metastasis by targeting ADAM9. miR-302a may serve as a potential therapeutic target for patients with OS.
\end{abstract}

Correspondence to: Professor Yan Cui, Department of Orthopedics, 89th Hospital of PLA, 256 Beigong West Road, Weifang, Shandong 261021, P.R. China

E-mail: yancui_89@163.com

Keywords: microRNA-302a, osteosarcoma, proliferation, migration, invasion, a disintegrin and metalloproteinase 9

\section{Introduction}

Osteosarcoma (OS) represents the most frequent malignant primary bone tumor arising from primitive bone-forming mesenchymal cells in children and adolescents (1). It occurs predominantly in metaphyseal regions of the long bone, with the distal femur and proximal tibia accounting for $\sim 50 \%$ of all OS cases (2). There is evidence that OS is caused by genetic and epigenetic changes, and environmental factors, which inhibit mesenchymal stem cells differentiating into osteoblasts (3). As a result of the progress made in diagnostic techniques, surgery, neoadjuvant radiotherapy and chemotherapy, the prognosis for patients with OS has improved (4). However, the presence of advanced phenotypes at the time of diagnosis, distant metastasis, and resistance to chemotherapy and/or therapy remain major causes of treatment failure (5). The molecular mechanisms underlying the initiation and progression of OS remain to be elucidated. Therefore, a comprehensive understanding of the carcinogenesis and progression of OS is required to investigate novel therapeutic strategies for this disease.

MicroRNAs (miRNAs) are an abundant group of endogenous, non-protein-coding, single stranded RNA molecules of $\sim 18-25$ nucleotides in length (6). miRNAs regulate gene expression at the transcriptional and post-transcriptional levels through direct interaction with the 3 'untranslated regions (3'UTRs) of their target genes via base-pairing, which leads to translational repression or mRNA degradation (7). Computational estimations suggest that there are $\sim 1,000$ miRNAs in the human genome, which regulate one third of human protein-encoding genes $(8,9)$. Through this mechanism, miRNAs are involved in the regulation of a wide range of physiological and pathological processes, including cell proliferation, cell cycle, apoptosis, survival, differentiation, invasion and metastasis (10-12). The dysregulation of miRNAs has been reported in several types of human cancer, in which these aberrantly expressed miRNAs are involved in tumor tumorigenesis, growth, metastasis, chemotherapy resistance and radiation resistance $(13,14)$. miRNAs can function either as tumor-suppressors or oncogenes in different types of human cancer depending on the characteristics of their target genes (15). Therefore, the identification of cancer-associated miRNAs may provide therapeutic targets for cancer.

The present study is the first, to the best of our knowledge, to report that miR-302a was significantly downregulated in OS tissues and cell lines. It also provided the first evidence 
that miR-302a targeted ADAM9 to inhibit cell proliferation, migration and invasion in OS.

\section{Materials and methods}

Patient samples. A total of 75 OS tissues and pair-matched non-tumorous tissues were collected from patients diagnosed with OS at the Department of Orthopedics, 89th Hospital of PLA (Weifang, China) between February 2013 and December 2015. No patients had received chemotherapy and/or radiotherapy prior to surgery. All specimens were frozen in liquid nitrogen and stored at $-80^{\circ} \mathrm{C}$. The present study was approved by the Ethics Committee of the 89 Hospital of PLA and written informed consent was also obtained from each patient.

Cell culture and transfection. OS cell lines (MG63, HOS, U2OS, SAOS-2) and a human normal osteoblastic cell line (hFOB 1.19) were purchased from America Type Culture Collection (Manassas, VA, USA), and grown in Dulbecco's modified Eagle's medium (Gibco; Thermo Fisher Scientific, Inc., Waltham, MA, USA) containing 10\% FBS (Gibco; Thermo Fisher Scientific, Inc.) and $1 \%$ penicillin/streptomycin in a $37^{\circ} \mathrm{C}$ humidified $5 \% \mathrm{CO}_{2}$ incubator. The hsa-miR-302a mimics and its negative control mimics (NC) were synthesized by GenePharma Co., Ltd. (Shanghai, China). ADAM9 small interfering (si)RNA and negative control siRNA (NC siRNA) were purchased from Guangzhou RiboBio Co., Ltd. (Guangzhou, China). The AMAM9 siRNA sequence was 5'-GGAATGGCATTGTGGGAA-3' and the NC siRNA sequence was 5'-TTCTCCGAACGTGTCACG TTT-3'. The cells were seeded in 6-well plates at a density of $8 \times 10^{5}$ cells/well, and transfected with oligonucleotides at room temperature using Lipofectamine 2000 (Invitrogen; Thermo Fisher Scientific, Inc.) when the cells were grown to $60-70 \%$ density.

RNA isolation and reverse transcription-quantitative polymerase chain reaction ( $R T-q P C R)$ analysis. RNA was isolated using TRIzol reagent (Invitrogen; Thermo Fisher Scientific, Inc.). The expression of miR-302a was detected using TaqMan microRNA assays (Applied Biosystems; Thermo Fisher Scientific, Inc.), with U6 as an internal control. To determine mRNA expression levels, total RNA was reverse transcribed into cDNA using an M-MLV Reverse Transcription system (Promega Corporation, Madison, WI, USA). SYBR Green PCR Master mix (Applied Biosystems; Thermo Fisher Scientific, Inc.) was used to measure the mRNA expression of ADAM9, and data was normalized to $\beta$-actin. This reaction included $2 \mu \mathrm{l}$ cDNA (100 ng), $2 \mu \mathrm{l}$ forward primer, $2 \mu \mathrm{l}$ reverse primer, $10 \mu \mathrm{l}$

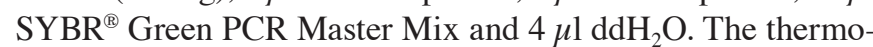
cycling conditions for qPCR were as follows: $95^{\circ} \mathrm{C}$ for $10 \mathrm{~min}$, followed by 40 cycles of $95^{\circ} \mathrm{C}$ for $15 \mathrm{sec}$ and $60^{\circ} \mathrm{C}$ for $1 \mathrm{~min}$. The primers were designed as follows: miR-302a, 5'-CGTGGA TGTACTTGCTTTGAA-3' (forward) and 5'-TCACCAAAA CATGGAAGCAC-3' (reverse); U6, 5'-GCTTCGGCAGCA CATATACTAAAAT-3' (forward) and 5'-CGCTTCACGAAT TTGCGTGTCAT-3' (reverse); ADAM9, 5'-CATTGAGGG AGGGACTTCTGGT-3' (forward) and 5'-ATGGGAACTGCT GAGGTTGCTT-3' (reverse); and $\beta$-actin, 5'-TGACGTGGA
CATCCGCAAAG-3' (forward) and 5'-CTGGAAGGTGGA CAGCGAGG-3' (reverse). The relative expression of miR-302a and ADAM9 were calculated using the $2^{-\triangle \Delta C q}$ method (16).

Cell proliferation assay. A Cell counting kit-8 (CCK-8; Dojindo Molecular Technologies, Inc., Kumamoto, Japan) assay was used to investigate the effect of miR-302a on OS cell proliferation. A total of 3,000 transfected OS cells were seeded into 96-well plates and incubated at $37^{\circ} \mathrm{C}$ in an atmosphere of $5 \% \mathrm{CO}_{2}$ for 1,2,3 and 4 days. At these time points, $10 \mu \mathrm{l}$ CCK-8 solution was added into each well and cultured for additional $2 \mathrm{~h}$. The absorbance at $450 \mathrm{~nm}$ was detected using an automatic multi-well spectrophotometer (Bio-Rad Laboratories, Inc., Hercules, CA, USA). Each assay was performed in triplicate and repeated three times.

Cell migration and invasion assay. Transwell chambers $(8-\mu \mathrm{m}$ pore size; BD Biosciences, San Jose, CA, USA), precoated with or without Matrigel (BD Biosciences) were used to the perform cell migration and invasion assays, respectively. In brief, a total of $3 \times 10^{4}$ transfected OS cells suspended in $200 \mu \mathrm{l}$ FBS-free culture medium were seeded in the upper Transwell chambers, and $500 \mu \mathrm{l}$ culture medium containing 10\% FBS was added to the lower chambers. Following incubation at $37^{\circ} \mathrm{C}$ in an atmosphere of $5 \% \mathrm{CO}_{2}$ for $48 \mathrm{~h}$, cotton swabs were used to remove the nonmigrated and noninvaded cells in the upper chamber. The migrated and invaded cells were fixed with $4 \%$ paraformaldehyde for $10 \mathrm{~min}$, stained with $0.5 \%$ crystal violet for $30 \mathrm{~min}$, and washed with PBS (Gibco; Thermo Fisher Scientific, Inc.). The cell numbers were counted in five randomly selected fields of each Transwell chambers under an inverted microscope (Olympus, Tokyo, Japan).

Luciferase reporter assay. The luciferase reporter vectors, pMir-ADAM9-3'UTR wild-type (WT) and pMirADAM9-3'UTR mutant-type (MUT), were synthesized by GenePharma, Co., Ltd. Using Lipofectamine 2000, HEK293T cells (American Type Culture Collection, Manassas, VA, USA) were transfected with luciferase reporter vectors, in addition to miR-302a mimics or NC. Following incubation for $48 \mathrm{~h}$ at $37^{\circ} \mathrm{C}$ in an atmosphere of $5 \% \mathrm{CO}_{2}$, luciferase activities were detected using a Dual-Luciferase Reporter Assay system (Promega Corporation). Renilla luciferase activities were normalized to firefly luciferase activities. Each assay was performed in triplicate.

Bioinformatics analysis. Bioinformatics analysis was conducted to predict the candidate genes of miR-302a using TargetScan (http://www.targetscan.org/) and miRanda (http://www.microrna.org/microrna/getDownloads.do).

Protein extraction and western blot analysis. Total protein was extracted using RIPA buffer (Beyotime Institute of Biotechnology, Haimen, China) supplemented with protease inhibitors and phosphate inhibitors. The concentration of total protein was detected using a BCA protein assay (Pierce; Thermo Fisher Scientific, Inc.). Equal quantities $(20 \mu \mathrm{g})$ of protein were separated by $10 \%$ sodium dodecyl sulfate-polyacrylamide gel electrophoresis and then transferred onto polyvinylidene difluoride membranes (EMD Millipore, Bedford, MA, USA). 
Subsequently, the membranes were blocked with 5\% non-fat skimmed milk powder in Tris-buffered saline (TBS), and incubated with mouse anti-human monoclonal ADAM9 antibody (1:1,000 dilution; cat. no. sc-377233; Santa Cruz Biotechnology, Inc., Santa Cruz, CA, USA) and mouse anti-human monoclonal GADPH antibody (1:1,000 dilution; cat. no. sc-166574; Santa Cruz Biotechnology, Inc.), at $4^{\circ} \mathrm{C}$ overnight. Following washing with TBS containing $0.5 \%$ Tween, the membranes were probed with goat anti-mouse horseradish peroxidase-conjugated secondary antibody (1:1,000 dilution; cat. no. sc-2005; Santa Cruz Biotechnology, Inc.) at room temperature for $1 \mathrm{~h}$. Protein bands were visualized using enhanced chemiluminescence, according to the manufacturer's protocol.

Statistical analysis. Data are presented as the mean \pm standard deviation. The differences between groupswere compared using Student's t-test or one-way analysis of variance using SPSS 13.0 statistical software (SPSS, Inc, Chicago, IL, USA). Student-Newman-Keuls test was utilized to compare between two groups in experiments involving multiple groups. $\mathrm{P}<0.05$ was considered to indicate a statistically significant difference.

\section{Results}

Expression of miR-302a in OS tissues and cell lines. The present study first investigated whether miR-302a was dysregulated in OS tissues, compared with pair-matched non-tumorous tissues, using RT-qPCR analysis. The results showed that the expression of miR-302a was significantly lower in the OS tissues, compared with the pair-matched non-tumorous tissues (Fig. 1A; $\mathrm{P}<0.05$ ).

RT-qPCR was also performed to detect the expression of miR-302a in OS cell lines, including the MG63, HOS, U2OS, SAOS-2 cell lines, and a human normal osteoblastic cell line (FOB 1.19). Comparison with the expression in the FOB 1.19 cells, the expression of miR-302a was significantly lower in all the examined OS cell lines (Fig. 1B; $\mathrm{P}<0.05$ ). These data suggested that miR-302a was downregulated in OS tissues and cell lines.

Downregulation of miR-302a is associated with aggressive tumor progression in patients with $O S$. The correlations between the expression of miR-302a and clinicopathological characteristics were analyzed. As shown in Table I, the expression levels of miR-302a were significantly correlated with tumor-node-metastasis $(\mathrm{TNM})$ stage $(\mathrm{P}=0.001)$ and metastasis $(\mathrm{P}=0.030)$ in $\mathrm{OS}$. However, no significant associations were found between the expression of miR-302a and the other clinicopathological characteristics, including age, gender, tumor size, and tumor location $(\mathrm{P}>0.05)$.

Expression of miR-302a in OS cells following transfection. To examine the functions of miR-302a in OS, the miR-302a mimics or NC mimic was injected into MG63 and U2OS cells, for relatively lower expression levels of miR-302a. Following transfection for $48 \mathrm{~h}$, RT-qPCR analysis was performed to evaluate its transfection efficiency. As shown in Fig. 2, miR-302a was significantly increased in the MG63 and U2OS cells transfected with miR-302a mimics $(\mathrm{P}<0.05)$.
Table I. Correlation between the expression of miR-302a and clinicopathological characteristics of in patients with osteosarcoma.

\begin{tabular}{|c|c|c|c|c|}
\hline \multirow{2}{*}{$\begin{array}{l}\text { Clinicopathological } \\
\text { feature }\end{array}$} & \multirow{2}{*}{$\begin{array}{l}\text { Cases } \\
\text { (n) }\end{array}$} & \multicolumn{2}{|c|}{$\begin{array}{l}\text { Expression } \\
\text { of } \mathrm{miR}-302 \mathrm{a}\end{array}$} & \multirow[b]{2}{*}{$\mathrm{P}$-value } \\
\hline & & Low & High & \\
\hline Age (years) & & & & 0.576 \\
\hline$<19$ & 55 & 29 & 26 & \\
\hline$\geq 19$ & 20 & 12 & 8 & \\
\hline Gender & & & & 0.213 \\
\hline Male & 39 & 24 & 15 & \\
\hline Female & 36 & 17 & 19 & \\
\hline Tumor size $(\mathrm{cm})$ & & & & 0.620 \\
\hline$<10$ & 31 & 18 & 13 & \\
\hline$\geq 10$ & 44 & 23 & 21 & \\
\hline Tumor location & & & & 0.837 \\
\hline Tibia/femur & 61 & 33 & 28 & \\
\hline Elsewhere & 14 & 8 & 6 & \\
\hline TNM stage & & & & 0.001 \\
\hline I-II & 35 & 12 & 23 & \\
\hline III & 40 & 29 & 11 & \\
\hline Metastasis & & & & 0.030 \\
\hline Present & 36 & 15 & 21 & \\
\hline Absent & 39 & 26 & 13 & \\
\hline
\end{tabular}

miR, microRNA; TNM, tumor-node-metastasis.
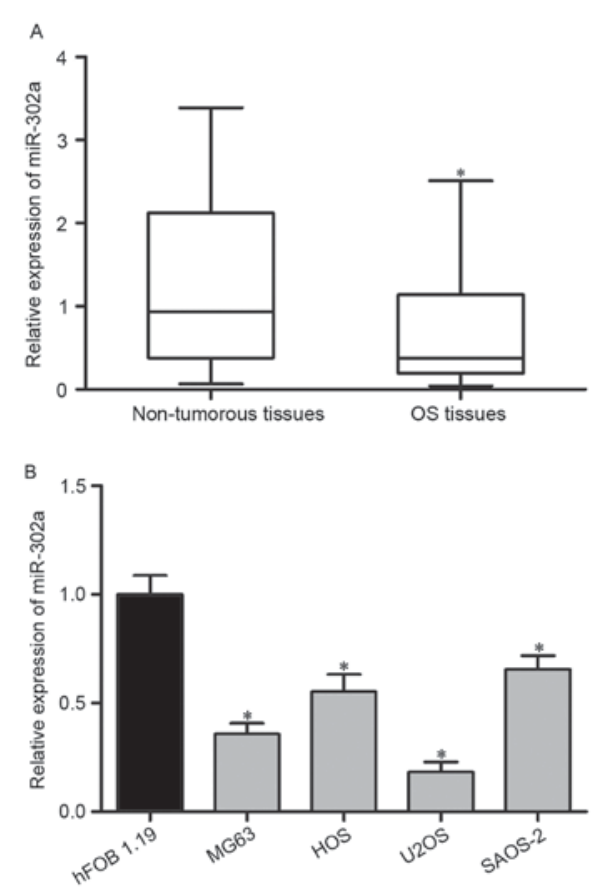

Figure 1. miR-302a is reduced in OS tissues and cell lines. (A) Expression levels of miR-302a in OS tissues were lower, compared with those in pair-matched non-tumorous tissues. (B) Expression level of miR-302a was reduced in MG63, HOS, U2OS, SAOS-2 OS cell lines, compared with the hFOB 1.19 human normal osteoblast cell line. "P<0.05, vs. hFOB 1.19. miR, microRNA; OS, osteosarcoma. 

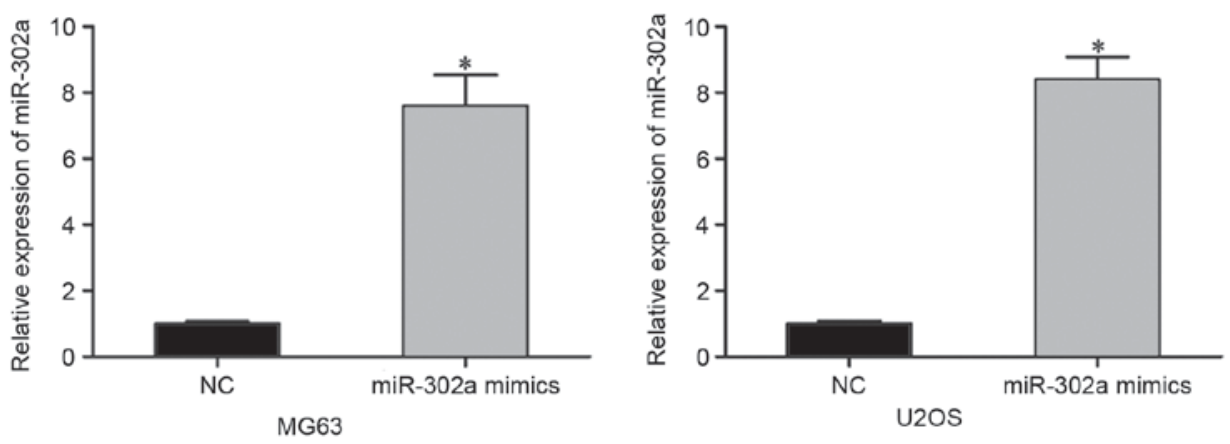

Figure 2. Expression of miR-302a following transfection with miR-3021 mimics. The expression of miR-302a was increased in the MG63 and U2OS cells following transfection with miR-302a mimics. "P<0.05, vs. NC. miR, microRNA; NC, negative control.
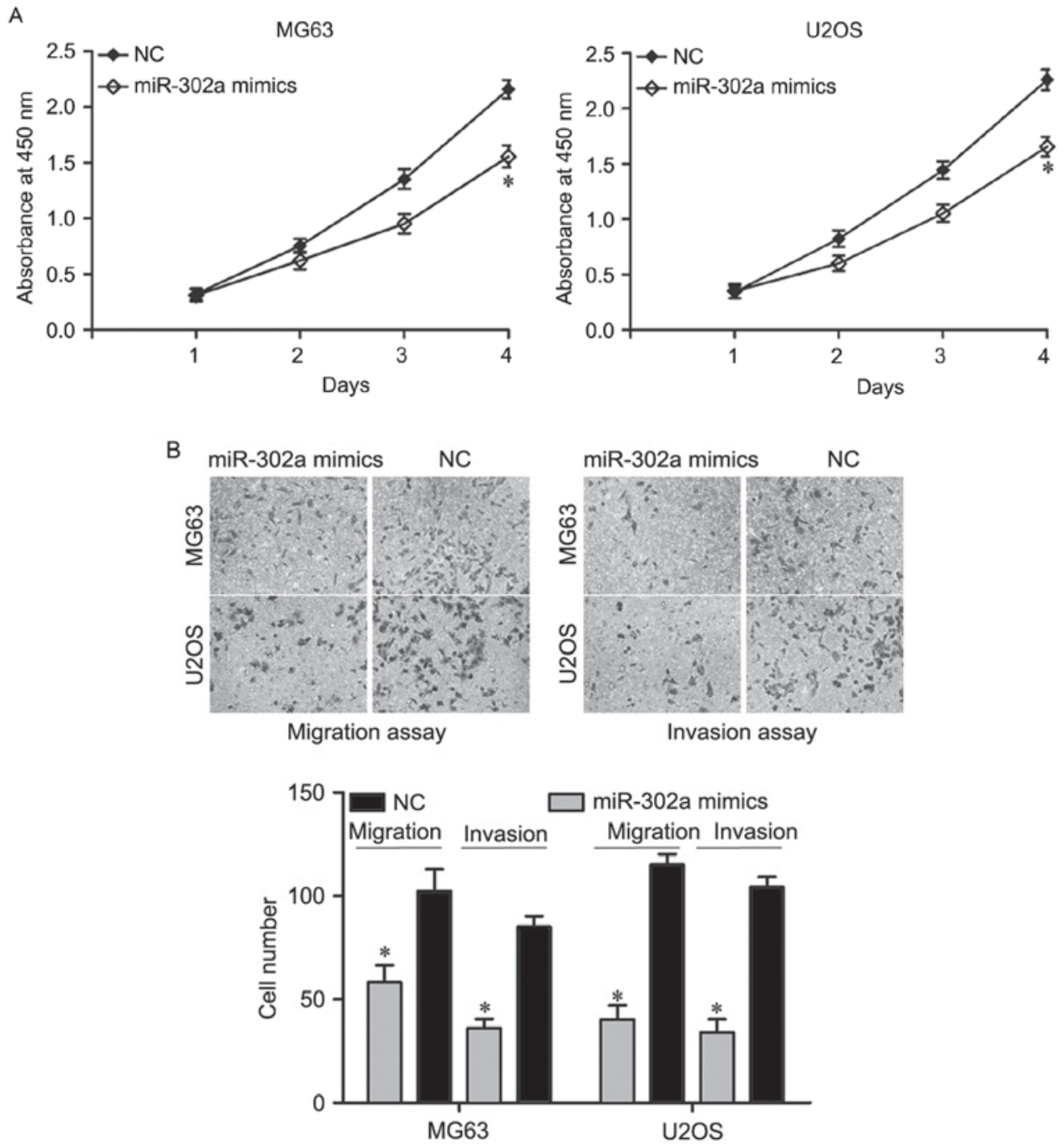

Figure 3. Overexpression of miR-302a inhibits MG63 and U2OS cell proliferation, migration and invasion. (A) Transfection with miR-302a mimics suppressed MG63 and U2OS cell proliferation. (B) Cell migration and invasion assays showed that the overexpression of miR-302a significantly decreased MG63 and U2OS cell migration and invasion (magnification, x200). ${ }^{*} \mathrm{P}<0.05$, vs. NC. miR, microRNA; NC, negative control.

Effects of miR-302a on the proliferation, migration and invasion of OS cells. To examine the effects of miR-302a on the proliferation of OS cells, cell proliferation assays were performed using the CCK-8 method. As shown in Fig. 3A, the overexpression of miR-302a inhibited MG63 and U2OS cell proliferation $(\mathrm{P}<0.05)$. As the expression level of miR-302a is correlated with metastasis in patients with OS, the present study examined whether ectopic miR-302a affected the migration and invasion capacities in OS. To confirm this hypothesis, cell migration and invasion assays were used, which revealed that the migration and invasion abilities were significantly decreased in the MG63 and U2OS cells transfected with 
A

\begin{tabular}{|c|c|c|}
\hline $\begin{array}{l}\text { ADAM9-3' UTR WT } \\
\text { Position 898-904 } \\
\text { hsa-miR-302a }\end{array}$ & $3^{\prime}$ & $\begin{array}{c}\text {...CUUGAGAAUUUCAUGAGCACUUU... } \\
\text { AGUGGUUUUGUACCUUCGUGAAU }\end{array}$ \\
\hline ADAM9-3' UTR MUT & 5 ' & ...CUUGAGAAUUUCAUGUCGUGAAL \\
\hline
\end{tabular}

B

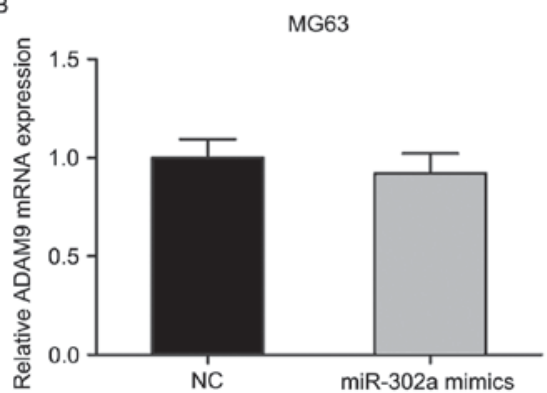

C

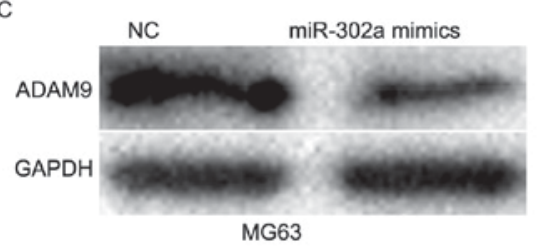

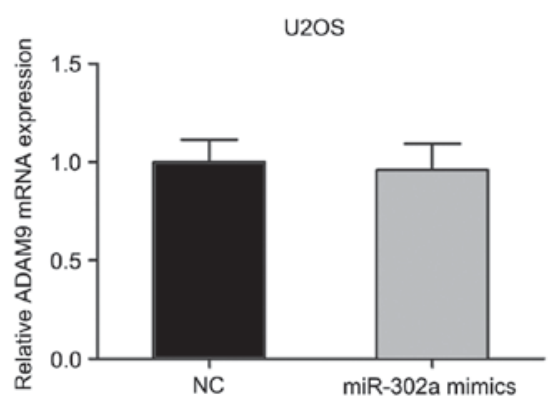

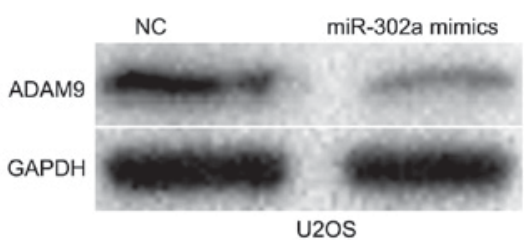

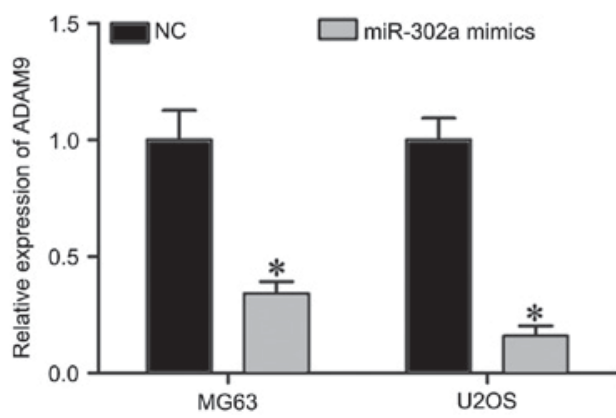

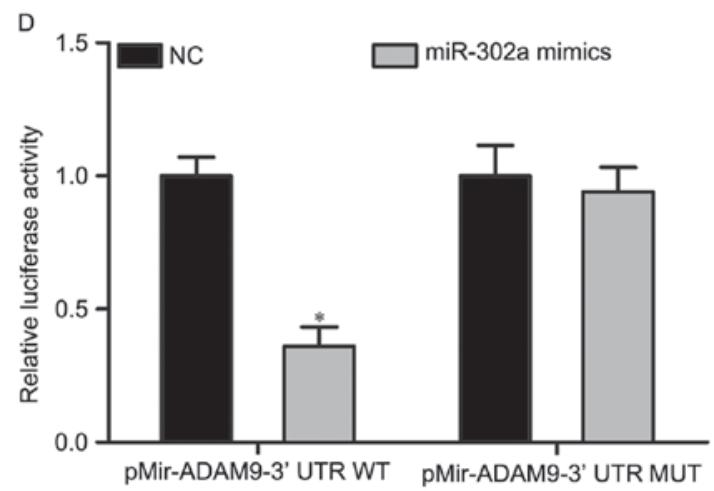

Figure 4. ADAM9 is a direct target gene of miR-302a. (A) Data from bioinformatics analysis indicating the putative target sites of miR-302a in the 3'UTR of ADAM9. (B) Reverse transcription-quantitative polymerase chain reaction analysis was performed to detect the mRNA levels of ADAM9 in MG63 and U2OS cells transfected with the miR-302a mimics or NC. (C) Western blot analysis was used to examine the protein level of ADAM9 in MG63 and U2OS cells injected with miR-302a mimics or NC. (D) Overexpression of miR-302a caused a significant decrease in luciferase activities of pMir-ADAM9-3'UTR WT, whereas co-transfection with pMir-ADAM9-3'UTR MUT and miR-302a mimics showed no difference, compared with the control group. * $<0.05$, vs. NC. miR, microRNA; 3'UTR, 3'untranslated region; ADAM9, a disintegrin and metalloproteinase 9; NC, negative control; WT, wild-type; MUT, mutant.

miR-302a mimics, compared with the cells transfected with NC (Fig. 3B; $\mathrm{P}<0.05$ ). These data suggested that miR-302a acted as a tumor suppressor in OS.

Identification of ADAM9 as a direct target of miR-302a. To investigate the molecular mechanism by which miR-302a inhibited OS cell growth and metastasis, bioinformatics analysis was performed using TargetScan (http://www.targetscan.org/) and miRanda (http://www. microrna.org/microrna/getDownloads.do). The analysis showed that ADAM9 was among the list of predicted miR-302a targets (Fig. 4A).

The correlation between miR-302a and ADAM9 was further examined by evaluating the mRNA and protein 

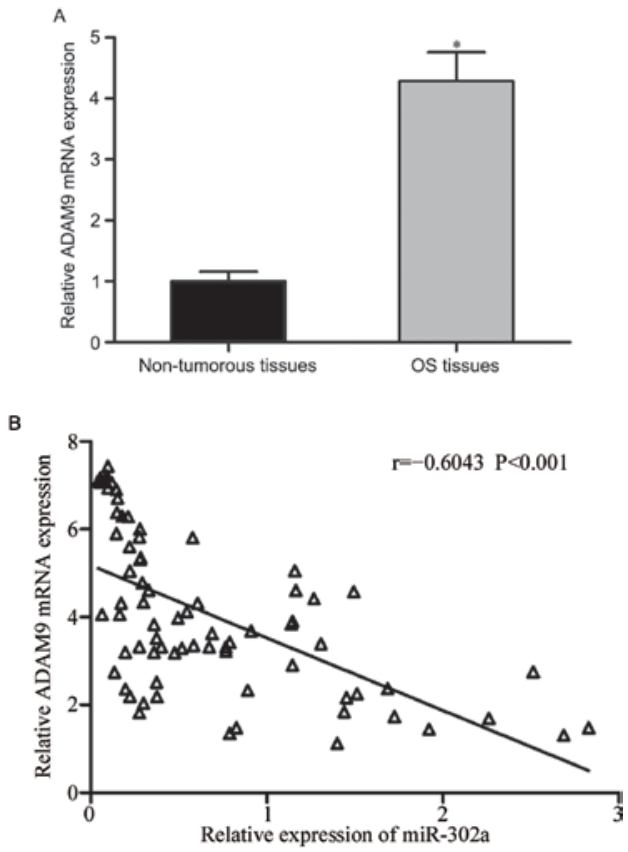

Figure 5. Expression of ADAM9 is inversely correlated with miR-302a in OS tissues. (A) Reverse transcription-quantitative polymerase chain reaction analysis was performed to determine the mRNA level of ADAM9 in OS tissues and pair-matched non-tumorous tissues. (B) Spearman's correlation analysis showed that the expression of ADAM9 was inversely correlated with that of miR-302a in OS tissues. " $\mathrm{P}<0.05$, vs. non-tumorous tissues. miR, microRNA; OS, osteosarcoma; ADAM9, a disintegrin and metalloproteinase 9 .

expression levels of ADAM9 in MG63 and U2OS cells following transfection with miR-302a mimics. The results of the RT-qPCR analysis showed that the restoration of miR-302a in MG63 and U2OS cells had no regulatory effect on the mRNA expression of ADAM9 (Fig. 4B; P>0.05). However, the results of western blot analysis revealed that the overexpression of miR-302a downregulated the protein expression of ADAM9 in MG63 and U2OS cells (Fig. 4C; $\mathrm{P}<0.05$ ). These results indicated that $\mathrm{miR}-302 \mathrm{a}$ regulated the expression of ADAM9 at the post-transcriptional level in OS.

Luciferase reporter assays were also performed to determine whether ADAM9 was a direct target gene of miR-302a. HEK293T cells were transfected with pMir-ADAM9-3'UTR WT or pMir-ADAM9-3'UTR MUT, in addition to miR-302a mimics or NC. As shown in Fig. 4D, the overexpression of miR-302a decreased the luciferase activity of pMir-ADAM9-3'UTR WT $(\mathrm{P}<0.05)$, but not that of pMir-ADAM9-3'UTR MUT. Taken together, these results demonstrated that ADAM9 was a direct target gene of miR-302a.

Expression of ADAM9 is inversely correlated with miR-302a in OS tissues. As ADAM9 was identified as a direct target gene of miR-302a in OS, the present study measured its expression in OS tissues and pair-matched non-tumorous tissues using RT-qPCR analysis. It was found that the mRNA levels of ADAM9 were significantly upregulated in OS tissues, compared with those in pair-matched non-tumorous tissues (Fig. 5A; P<0.05). Spearman's correlation analysis revealed that ADAM9 was inversely correlated with the expression of miR-302a in OS tissues (Fig. 5B; r=-0.6043; $\mathrm{P}<0.001$ ).
Knockdown of ADAM9 inhibits cell proliferation, migration and invasion in OS. To investigate the roles of ADAM9 in OS, loss-of-function experiments were performed. MG63 and U2OS cells were transfected with ADAM9 siRNA or NC siRNA. As shown in Fig. 6A, ADAM9 siRNA markedly decreased the expression of ADAM9 in MG63 and U2OS cells $(\mathrm{P}<0.05)$. Following transfection, cell proliferation, cell migration and invasion assays were performed, which demonstrated that the knockdown of ADAM9 significantly suppressed MG63 and U2OS cell proliferation (Fig. 5B; $\mathrm{P}<0.05$ ), migration and invasion (Fig. 5C; $\mathrm{P}<0.05$ ). The results showed that the effects of ADAM9 knockdown were similar with those of miR-302a in OS cells, suggesting that ADAM9 was a direct and functional target of miR-302a in OS.

\section{Discussion}

Previous studies have provided evidence for the dysregulation of miR-302a in various types of human cancer. For example, in colorectal cancer, the expression of miR-302a was found to be lower in tumor cell lines, compared with that in normal colon epithelium cells (17). Zhang et al (18) reported that, compared with normal prostate tissues, the expression level of miR-302a was reduced in prostate cancer tissues, and even lower in tumor tissues with a Gleason score $\geq 8$. Liang et al $(19,20)$ demonstrated that miR-302a was significantly downregulated in metastatic breast cancer tissues and cell lines, and in irradiated breast cancer cell lines. Guo et al (21) showed that miR-302a was significantly downregulated in ovarian cancer tissues and cell lines, compared with normal cervical tissues and normal cells, respectively. The reduced expression levels of miR-302a in tumor tissues has also been shown to correlate with TNM stage in patients with ovarian cancer (21). However, until now, there have been no reports on the expression level of miR-302a in OS. The present study initially measured the expression of miR-302a in OS tissues and pair-matched non-tumorous tissues using RT-qPCR analysis. The results showed that miR-302a was markedly downregulated in OS tissues. Consistent with the expression pattern in tissues, OS cell lines also showed reduced expression of miR-302a compared with the human normal osteoblastic cell line. The statistical analysis showed that low expression levels of miR-302a were correlated with TNM stage and metastasis in patients with OS. These data suggested that miR-302a may be involved in the progression of several types of human cancer.

Previous studies have reported significant roles of miR-302a in cancer progression. Wei et al (17) found that the overexpression of miR-302a suppressed cell proliferation and invasion in colorectal cancer. In prostate cancer, functional investigations revealed that the restoration of miR-302a inhibited prostate cancer cells growth in vitro and in vivo, and enhanced G1/S cell cycle arrest (18). In breast cancer, the upregulation of miR-302a decreased tumor cell invasion abilities and metastasis in vitro and in vivo (19). In addition, the ectopic expression of miR-302a improved the radiosensitivity of breast cancer cells to radiation therapy in vitro and in vivo (20). In ovarian cancer, the induced expression of miR-302a has been found to repress cell proliferation, induce cell cycle arrest at the 

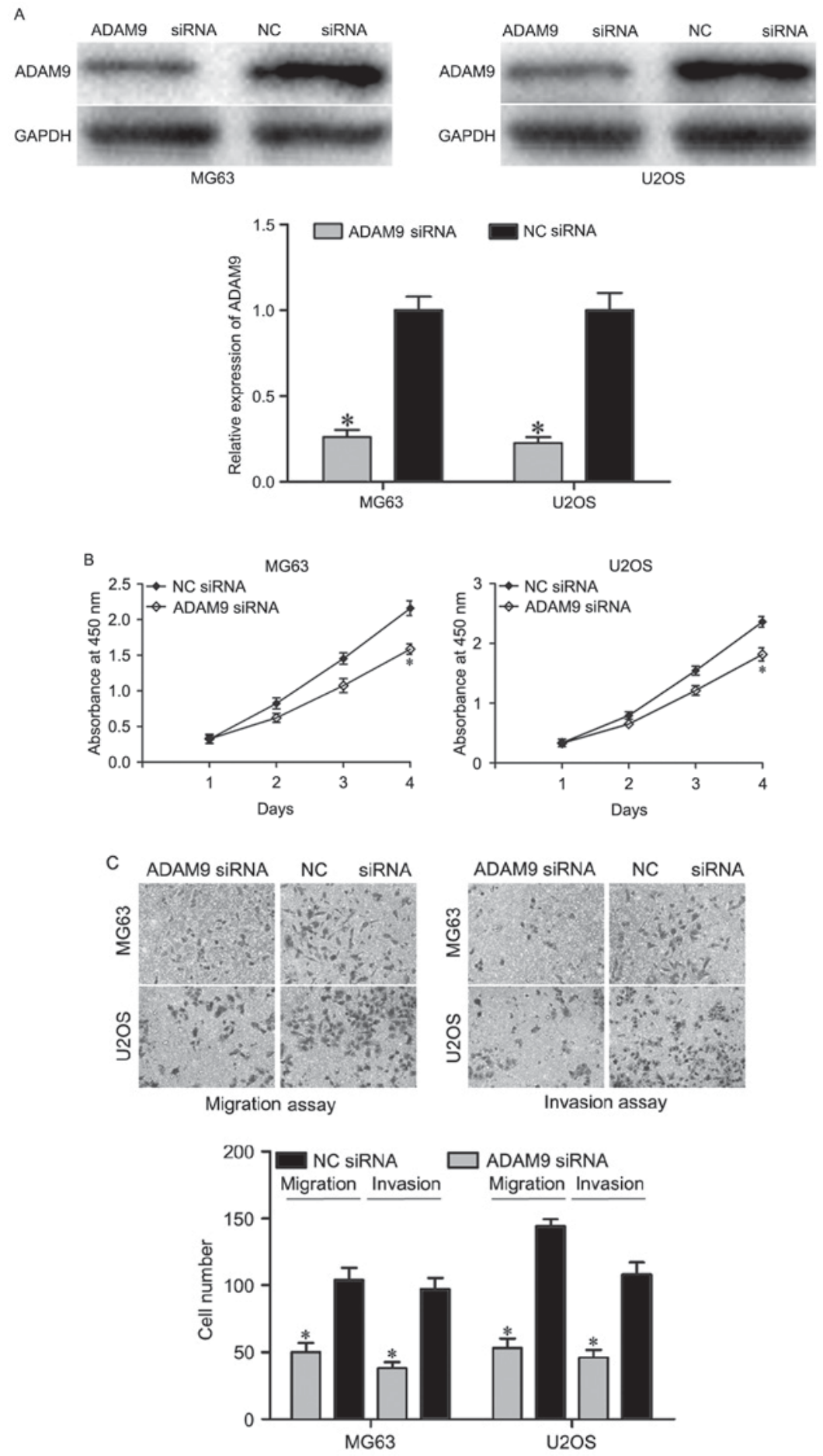

Figure 6. ADAM9 knockdown inhibits MG63 and U2OS cell proliferation, migration and invasion. (A) ADAM9 was downregulated in MG63 and U2OS cells following transfection with ADAM9 siRNA. (B) Transfection of ADAM9 siRNA decreased MG63 and U2OS cell proliferation. (C) Cell migration and invasion assays showed that the knockdown of ADAM9 suppressed MG63 and U2OS cell migration and invasion abilities. "P<0.05, vs. NC siRNA. ADAM9, a disintegrin and metalloproteinase 9; siRNA, small interfering RNA; NC, negative control.

G1 phase and decrease transition from the G1 phase to the $\mathrm{S}$ phase (21). However, the functions of miR-302 in OS have not been reported previously. In the present study, the downregulation of miR-302a in OS suggested that miR-302a may act as a potential tumor suppressor in OS. Functional experiments showed that the overexpression of miR-302a suppressed OS cell proliferation, migration and invasion in vitro, suggesting that the decreased expression of miR-302a in OS may promote OS cell growth and metastasis.
At the molecular level, several target genes of miR-302a have been identified, including mitogen-activated protein kinase (MAPK) in colorectal cancer (17), AKT in prostate cancer (18), C-X-C chemokine receptor type 4 (19), breast cancer resistance protein (22), MAPK kinase 1 (23) in breast cancer, and syndecan-1 in ovarian cancer (21). In the present study, the results demonstrated that ADAM9 may be one of the direct targets of miR-302a in OS. Members of the ADAM family have been demonstrated to contribute to several 
biological functions, including fertilization, adhesion, migration and proteolysis $(24,25)$. ADAM9, a member of the ADAM family, was found to be significantly upregulated in non-small cell lung cancer (26), colon cancer (27), gastric cancer (28) and prostate cancer (29). Several studies have reported that ADAM9 is important in cancer cell growth, invasion and metastasis (28,30-32). A study by Jiang et al (33) found that ADAM9 is produced and secreted by human osteoblasts and acts as one of the insulin-like growth factor binding protein-5 proteases, which is important in regulating bone formation. ADAM9 was also reported to be upregulated in OS tissues and cell lines, compared with normal tissues and cells (33). These findings indicated that ADAM9 may be involved in the initiation and progression of OS, and may be investigated as an effective therapeutic target for OS.

In conclusion, the present study showed that miR-302a was significantly downregulated in OS. Its reduced expression was correlated with TNM stage and metastasis in OS. Functionally, the upregulation of miR-302a inhibited the proliferation, migration and invasion of OS cells by directly targeting ADAM9, thus being involved in the carcinogenesis and progression of OS. The results of the present study suggested that miR-302a may be a therapeutic target in the treatment of patients with OS.

\section{References}

1. Ando K, Heymann MF, Stresing V, Mori K, Rédini F and Heymann D: Current therapeutic strategies and novel approaches in osteosarcoma. Cancers (Basel) 5: 591-616, 2013.

2. He H, Ni J and Huang J: Molecular mechanisms of chemoresistance in osteosarcoma (Review). Oncol Lett 7: 1352-1362, 2014.

3. Sun L, Li Y, Zhang J, Li H, Li B and Ye Z: Prognostic value of pathologic fracture in patients with high grade localized osteosarcoma: A systemic review and meta-analysis of cohort studies. J Orthop Res 33: 131-139, 2015.

4. Broadhead ML, Clark JC, Myers DE, Dass CR and Choong PF: The molecular pathogenesis of osteosarcoma: A review. Sarcoma 2011: 959248, 2011.

5. Poletajew S, Fus L and Wasiutynski A: Current concepts on pathogenesis and biology of metastatic osteosarcoma tumors. Ortop Traumatol Rehabil 13: 537-545, 2011 (In English, Polish).

6. Carrington JC and Ambros V: Role of microRNAs in plant and animal development. Science 301: 336-338, 2003.

7. Liu J: Control of protein synthesis and mRNA degradation by microRNAs. Curr Opin Cell Biol 20: 214-221, 2008.

8. Nouraee $\mathrm{N}$ and Mowla SJ: miRNA therapeutics in cardiovascular diseases: Promises and problems. Front Genet 6: 232, 2015.

9. Bertoli G, Cava C and Castiglioni I: MicroRNAs: New biomarkers for diagnosis, prognosis, therapy prediction and therapeutic tools for breast cancer. Theranostics 5: 1122-1143, 2015.

10. Aigner A: MicroRNAs (miRNAs) in cancer invasion and metastasis: Therapeutic approaches based on metastasis-related miRNAs. J Mol Med (Berl) 89: 445-457, 2011.

11. Rottiers V and Näär AM: MicroRNAs in metabolism and metabolic disorders. Nat Rev Mol Cell Biol 13: 239-250, 2012.

12. Cho WC: MicroRNAs: Potential biomarkers for cancer diagnosis, prognosis and targets for therapy. Int J Biochem Cell Biol 42: $1273-1281,2010$

13. Ueda T, Volinia S, Okumura H, Shimizu M, Taccioli C, Rossi S, Alder H, Liu CG, Oue N, Yasui W, et al: Relation between microRNA expression and progression and prognosis of gastric cancer: A microRNA expression analysis. Lancet Oncol 11: $136-146,2010$
14. Chistiakov DA and Chekhonin VP: Contribution of microRNAs to radio- and chemoresistance of brain tumors and their therapeutic potential. Eur J Pharmacol 684: 8-18, 2012.

15. Jones KB, Salah Z, Del Mare S, Galasso M, Gaudio E, Nuovo GJ, Lovat F, LeBlanc K, Palatini J, Randall RL, et al: miRNA signatures associate with pathogenesis and progression of osteosarcoma. Cancer Res 72: 1865-1877, 2012.

16. Fan W, Huang J, Xiao H and Liang Z: MicroRNA-22 is downregulated in clear cell renal cell carcinoma, and inhibits cell growth, migration and invasion by targeting PTEN. Mol Med Rep 13: 4800-4806, 2016.

17. Wei ZJ, Tao ML, Zhang W, Han GD, Zhu ZC, Miao ZG, Li JY and Qiao ZB: Up-regulation of microRNA-302a inhibited the proliferation and invasion of colorectal cancer cells by regulation of the MAPK and PI3K/Akt signaling pathways. Int J Clin Exp Pathol 8: 4481-4491, 2015.

18. Zhang GM, Bao CY, Wan FN, Cao DL, Qin XJ, Zhang HL, Zhu Y, Dai B, Shi GH and Ye DW: MicroRNA-302a suppresses tumor cell proliferation by inhibiting AKT in prostate cancer. PLoS One 10: e0124410, 2015.

19. Liang Z, Bian X and Shim H: Inhibition of breast cancer metastasis with microRNA-302a by downregulation of CXCR4 expression. Breast Cancer Res Treat 146: 535-542, 2014.

20. Liang Z, Ahn J, Guo D, Votaw JR and Shim H: MicroRNA-302 replacement therapy sensitizes breast cancer cells to ionizing radiation. Pharm Res 30: 1008-1016, 2013.

21. Guo T, Yu W, Lv S, Zhang C and Tian Y: MiR-302a inhibits the tumorigenicity of ovarian cancer cells by suppression of SDC1. Int J Clin Exp Pathol 8: 4869-4880, 2015.

22. Wang Y, Zhao L, Xiao Q, Jiang L, He M, Bai X, Ma M, Jiao X and Wei M: miR-302a/b/c/d cooperatively inhibit BCRP expression to increase drug sensitivity in breast cancer cells. Gynecol Oncol 141: 592-601, 2016.

23. Zhao L, Wang Y, Jiang L, He M, Bai X, Yu L and Wei M: MiR-302a/b/c/d cooperatively sensitizes breast cancer cells to adriamycin via suppressing P-glycoprotein (P-gp) by targeting MAP/ERK kinase kinase 1 (MEKK1). J Exp Clin Cancer Res 35: 25, 2016.

24. Blobel CP: ADAMs: Key components in EGFR signalling and development. Nat Rev Mol Cell Biol 6: 32-43, 2005.

25. Edwards DR, Handsley MM and Pennington CJ: The ADAM metalloproteinases. Mol Aspects Med 29: 258-289, 2008.

26. Zhang J, Qi J, Chen N, Fu W, Zhou B and He A: High expression of a disintegrin and metalloproteinase- 9 predicts a shortened survival time in completely resected stage I non-small cell lung cancer. Oncol Lett 5: 1461-1466, 2013.

27. Li J, Ji Z, Qiao C, Qi Y and Shi W: Overexpression of ADAM9 promotes colon cancer cells invasion. J Invest Surg 26: 127-133, 2013.

28. Kim JM, Jeung HC, Rha SY, Yu EJ, Kim TS, Shin YK, Zhang X, Park KH, Park SW, Chung HC and Powis G: The effect of disintegrin-metalloproteinase ADAM9 in gastric cancer progression. Mol Cancer Ther 13: 3074-3085, 2014.

29. Sung SY, Kubo H, Shigemura K, Arnold RS, Logani S, Wang R, Konaka H, Nakagawa M, Mousses S, Amin M, et al: Oxidative stress induces ADAM9 protein expression in human prostate cancer cells. Cancer Res 66: 9519-9526, 2006.

30. Chang L, Gong F and Cui Y: RNAi-mediated A disintegrin and metalloproteinase 9 gene silencing inhibits the tumor growth of non-small lung cancer in vitro and in vivo. Mol Med Rep 12: 1197-1204, 2015.

31. Martin AC, Cardoso AC, Selistre-de-Araujo HS and Cominetti MR: Recombinant disintegrin domain of human ADAM9 inhibits migration and invasion of DU145 prostate tumor cells. Cell Adh Migr 9: 293-299, 2015.

32. Chen CM, Hsieh YH, Hwang JM, Jan HJ, Hsieh SC, Lin SH and Lai CY: Fisetin suppresses ADAM9 expression and inhibits invasion of glioma cancer cells through increased phosphorylation of ERK1/2. Tumour Biol 36: 3407-3415, 2015.

33. Jiang L, He A, Zhang Q and Tao C: miR-126 inhibits cell growth, invasion, and migration of osteosarcoma cells by downregulating ADAM-9. Tumour Biol 35: 12645-12654, 2014. 